\title{
Partie générale de l'assurance sociale: moins de problèmes d'encaissement liés au régime du tiers garant
}

Robert Gmür, Service juridique de la FMH

La loi fédérale sur la partie générale du droit des assurances sociales (LPGA) est entrée en vigueur le $1^{\text {er }}$ janvier 2003. A la même date, le Conseil fédéral a édicté une ordonnance s'y rapportant (OPGA) et adapté de nombreuses dispositions figurant dans l'Ordonnance sur l'assurancemaladie (OAMal).

\section{Aperçu général}

Commençons par le plus important: La LPGA fait totalement abstraction de ce qu'on appelle le droit médical, à savoir de la législation concernant les contrats tarifaires et l'admission des médecins à pratiquer à la charge de l'assurance sociale. Aucun changement, donc, en ce qui concerne ce point d'une importance essentielle pour le corps médical.

Après quinze ans de débats et non sans quelques difficultés, le Parlement a finalement adopté une version «allégée» de la LPGA, moins ambitieuse que le projet initial. Celle-ci s'applique à toutes les branches des assurances sociales, à l'exception de la prévoyance professionnelle. Les principales nouveautés sont les suivantes:

- Les assurés ont le droit d'être renseignés et conseillés par les organes des diverses assurances sociales, un principe qui n'était ancré jusqu'ici que dans l'assurance-maladie.

- Une procédure administrative unifiée est désormais applicable. Les assurés peuvent faire opposition, dans tous les secteurs de l'assurance sociale, aux décisions rendues par les assureurs, ce que seule l'assurance-maladie et accident prévoyait jusqu'à présent. Pour l'assuré, faire opposition signifie préciser une fois encore son point de vue auprès de l'assureur sans intenter immédiatement une action en justice. Cette étape supplémentaire peut toutefois présenter le désavantage de rendre plus longue encore une procédure qui l'est déjà suffisamment, ce qui pose problème par exemple dans le cas de l'AI. Seule l'expé- rience permettra de porter un jugement sur l'impact concret et les avantages et inconvénients de ce changement.

- Les litiges entre assureurs et assurés portant sur des prestations peuvent dorénavant être réglés par accord ou, pour reprendre le terme de la loi, par «transaction». Toutefois, même dans le cadre d'une telle transaction, il ne peut être convenu de prestations non admises par la loi. L'OFAS a annoncé son intention d'élaborer des lignes directrices en la matière.

- La LPGA améliore la coordination des droits de l'assuré, que ce soit entre les assureurs sociaux ou par rapport aux tiers responsables. La surindemnisation est à présent partout interdite et la loi stipule, dans le cadre d'un système global, quelle assurance sociale est tenue, en cas de doute, d'assurer la prise en charge provisoire des prestations.

- Enfin, la LPGA introduit, quelque peu timidement, une obligation de verser des intérêts moratoires: l'assuré y a droit lorsque les prestations lui sont payées avec plus de deux ans de retard sans qu'il y ait de sa faute.

Le lecteur désireux d'étudier ces questions en profondeur consultera avec profit la revue «Sécurité sociale» 5/2002 [1] publiée par l'OFAS, spécialement consacrée à cette nouvelle loi.

Comme nous l'avons déjà relevé, la LPGA met l'accent sur le droit procédural. Toutefois, certaines des modifications qu'elle introduit peuvent se révéler intéressantes, d'un point de vue pratique, pour le corps médical:

\section{Encaissement de créances d'honoraires: I'incessibilité du droit aux prestations, cette pomme de discorde}

On ne connaît que trop bien la situation prévalant actuellement: presque toutes les caisses maladie précisent, dans leurs statuts ou leurs conditions générales, que l'assuré ne peut céder son 
2 Voir aussi Duc J-L. De la cession du droit aux prestations dans l'assurance-maladie obligatoire. SZS/RSAS 2002;46:276.

* En cas de besoin, vous trouverez un texte-type sous www.fmh.ch $\rightarrow$ droit $\rightarrow$ documents types. droit au remboursement des prestations, pas même à son médecin. Certes, dans certains cantons, des exceptions sont possibles par le biais de réglementations plus ou moins explicites, voire de gentlemen's agreements. Mais ces dispositions s'avèrent souvent difficiles à mettre en pratique, et leur exécution requiert autant de temps qu'elle suscite de contrariétés.

La nouvelle LPGA vient clarifier cet aspect: si elle étend l'interdiction de céder le droit aux prestations - réglementée jusqu'à maintenant dans le cadre de la seule AVS - à toutes les branches de l'assurance sociale (art. 22 LPGA), une exception est explicitement ménagée à l'art. 42, al. 1 de la LAMal. Celui-ci est ainsi libellé: En dérogation à l'art. 22, al. 1, LPGA, ce droit peut être cédé au fournisseur de prestations.

Le Parlement a pris cette décision en toute connaissance de cause: l'incessibilité de ce droit signifierait en effet «que le patient ne pourrait plus céder au médecin ses prétentions vis-à-vis de l'assurance-maladie. C'est pourquoi une dérogation à l'interdiction de cession est introduite dans l'art. 42 al. 1 LAMal, en faveur des fournisseurs de prestations en général.» (Feuille fédérale 1999, no 23, p. 4218). Cette disposition s'explique par la nécessité d'assurer la continuité de la prise en charge médicale des patients confrontés à des problèmes financiers, sociaux ou médicaux (de dépendance, par exemple). En outre, elle offre aussi - en des temps où l'éthique en matière de paiements est des moins en moins respectée - une certaine garantie pour le recouvre- ment de créances auprès de mauvais payeurs notoires, garantie toutefois limitée si l'on songe à l'ingéniosité de certains patients en la matière ...

La nouvelle législation implique, pour les assureurs-maladies, qu'ils devront modifier leurs statuts ou leurs conditions générales et accepter, à l'avenir, les déclarations de cession dans le domaine de l'assurance de base obligatoire [2]. Le danger, évoqué par certains, de voir les médecins mésuser de ce nouvel instrument et «miner» ainsi le système du tiers garant, n'est certainement pas à craindre: non seulement la procédure administrative est-elle compliquée (préparation et envoi de la déclaration de cession*, modification du fichier de base, etc.), mais un médecin ne peut pas se permettre de brusquer des patients - par exemple lorsqu'ils lui sont inconnus - sans discernement ni réflexion. Dans son propre intérêt, il ne recourra à la cession du droit aux prestations d'assurance que dans des cas précis et justifiés.

Reste ouverte la question de savoir si le patient peut céder son droit au remboursement de manière complète, sans déduction de la participation et de la franchise, ou si le médecin devra se contenter $d u$ «montant net» de la prestation d'assurance. Dans ce dernier cas et si le patient a convenu d'une franchise élevée, le but visé par le Parlement, à savoir assurer la prise en charge médicale, ne pourrait être atteint. Si les caissesmaladie ne parvenaient pas à proposer une solution raisonnable sur ce point, nul doute que le dernier mot reviendrait alors aux tribunaux. 